\title{
Calculation of the Universal Gravitational Constant, of the Hubble Constant, and of the Average CMB Temperature*
}

\author{
Claude Mercier \\ Independent Researcher, Baie-Comeau, Canada \\ Email: claude.mercier@gctda.com
}

How to cite this paper: Mercier, C. (2019) Calculation of the Universal Gravitational Constant, of the Hubble Constant, and of the Average CMB Temperature. Journal of Modern Physics, 10, 641-662.

https://doi.org/10.4236/jmp.2019.106046

Received: April 30, 2019

Accepted: May 21, 2019

Published: May 24, 2019

Copyright $\odot 2019$ by author(s) and Scientific Research Publishing Inc. This work is licensed under the Creative Commons Attribution International License (CC BY 4.0).

http://creativecommons.org/licenses/by/4.0/ (c) (i) Open Access

\begin{abstract}
Dirac made the hypothesis that all large, dimensionless numbers that could be constructed from the important natural units of cosmology and atomic theory were connected [1] [2]. Although Dirac did not succeed in exactly matching all these numbers, he suspected that there was a way to unify all of them. Dirac's hypothesis leads to the $N$ constant which unifies most of physics' parameters. It represents the maximum number of photons with a wavelength equal to the universe circumference. Using a new cosmological model, we found the $\beta$ constant which represents the ratio between the expansion speed of matter in the universe and the speed of light. With these constants, we can now calculate accurately several physics parameters, including the universal gravitational constant $G$, the Hubble constant $H_{0}$, and the average temperature $T$ of the cosmological microwave background (CMB). Our equations show that $G, H_{0}$ and $T$ are not really constant over space and time.
\end{abstract}

$$
\begin{gathered}
G \approx 6.673229809(86) \times 10^{-11} \mathrm{~m}^{3} \cdot \mathrm{kg}^{-1} \cdot \mathrm{s}^{-2}, T \approx 2.7367958(16) \mathrm{K} \\
H_{0} \approx 72.09548580(32) \mathrm{km} \cdot \mathrm{s}^{-1} \cdot \mathrm{MParsec}^{-1}, \quad N \approx 6.303419702(84) \times 10^{121}
\end{gathered}
$$

\section{Keywords}

Gravitational Constant, Hubble, Dirac Large Numbers Hypothesis, $\mathrm{CMB} / \mathrm{CMBR}$

\section{Introduction}

The universal gravitational constant $G$, the Hubble constant $H_{0}$ and the average temperature $T$ of the cosmological microwave background (CMB) of the un-

${ }^{*}$ Calculation of $G, H_{0}$, and $T$. 
iverse suffer from higher uncertainties than most of other constants because, for the moment, they are only measured. The measurement of $G$ is imprecise because of the low intensity of gravitational forces. Even with the best torsion scale, collected data are tainted by errors caused by the influence of masses that circulate in the vicinity (Sun, Moon, Earth, etc.). The $H_{0}$ constant is measured using observations of distant galaxies and the results vary according to the method used. With respect to the average temperature of the diffuse background of the $T$ universe, it is difficult to make an accurate and reliable measurement of its value, especially near absolute zero $(0 \mathrm{~K})$.

The purpose of this paper is to provide to the modern metrology system new equations that could accurately determine $G, H_{0}, T$, and $N$ from other well-defined constants. The value of $N$ represents the maximum number of photons existing in the universe which have the lowest energy possible (with a wavelength equal to the circumference of the universe). These equations may help to discriminate different measurement results and identify the best methods to use. Several measurements are displayed as having incredibly low tolerances but do not overlap with other measurements that display similar tolerances.

We will begin by defining the parameters used in our article while giving their value using CODATA 2014 [3]. Subsequently, in order to find the desired equations, we will show our model of the universe that will, among other things, allow finding the $\beta$ constant. The value of $\beta$ represents the ratio between the expansion speed of the material universe and the expansion speed of the luminous universe (which is, for now, the speed of light in vacuum $c$ ). This constant is crucial in the subsequent calculations of several physics' constants. A modified version of Dirac's hypothesis on large numbers will be presented in order to establish a link with the fine-structure constant $\alpha$. Using this link, we will find equations giving the exact values of $H_{0}$ and $G$ while explaining why these parameters of the universe are not constant through space and time. What we will end up with is a modified version of the Weinberg equation that will provide a tool to "measure" the Hubble constant $H_{0}$ as a function of the universal gravitational constant $G$. Through this article, several links are made between the infinitely small and the infinitely large.

\section{Development}

\subsection{Values of Physics Parameters}

In general, we will use the concise form of notation to display tolerances. For example, typically, 2.736(17) K will mean $2.736 \pm 0.017 \mathrm{~K}$.

According to Salvatelli, Hubble constant is $H_{0} \approx 72.1_{-2.3}^{+3.2} \mathrm{~km} \cdot \mathrm{s}^{-1} \cdot \mathrm{MParsec}^{-1}$ [4]. This value will be used until we get a precise value of $H_{0}$ from Equation (44) and Equation (63). It should be noted that $1 \mathrm{MParsec} \approx 3.085677581 \times 10^{22} \mathrm{~m}$.

The average temperature of the Cosmic Microwave Background (CMB) has been measured by the Cobra probe at $T \approx 2.736(17) \mathrm{K}$ [5]. According to Fixsen, it is $T \approx 2.72548(57) \mathrm{K}[6]$. 
According to the CODATA 2014 [3], the physics parameters used are:

- Speed of light in vacuum $c \approx 299792458 \mathrm{~m} \cdot \mathrm{s}^{-1}$

- Electric constant in vacuum $\varepsilon_{0} \approx 8.854187817 \times 10^{-12} \mathrm{~F} \cdot \mathrm{m}^{-1}$

- Magnetic constant in vacuum $m_{0} \approx 4 \pi \times 10^{8} \mathrm{~N} \cdot \mathrm{A}^{-2}$

- Planck constant $h \approx 6.626070040(81) \times 10^{-34} \mathrm{~J} \cdot \mathrm{s}$

- Planck length $L_{p} \approx 1.616229(38) \times 10^{-35} \mathrm{~m}$

- Planck time $t_{p} \approx 5.39116(13) \times 10^{-44} \mathrm{~s}$

- Planck mass $m_{p} \approx 2.176470(51) \times 10^{-8} \mathrm{~kg}$

- Planck temperature $T_{p} \approx 1.416808(33) \times 10^{35} \mathrm{~K}$

- Universal gravitational constant $G \approx 6.67408(31) \times 10^{-11} \mathrm{~m}^{3} \cdot \mathrm{kg}^{-1} \cdot \mathrm{s}^{-2}$

- Electron charge $q_{e} \approx 1.6021766208(98) \times 10^{-19} \mathrm{C}$

- Electron mass $m_{e} \approx 9.10938356(11) \times 10^{-31} \mathrm{~kg}$

- Classical electron radius $r_{e} \approx 2.8179403227(19) \times 10^{-15} \mathrm{~m}$

- Fine-structure constant $\alpha \approx 7.2973525664(17) \times 10^{-3}$

- Boltzmann constant $k_{b} \approx 1.38064852(79) \times 10^{-23} \mathrm{~J} \cdot \mathrm{K}^{-1}$

- Stephen-Boltzmann constant $\sigma \approx 5.670367(13) \times 10^{-8} \mathrm{~W} \cdot \mathrm{m}^{-2} \cdot \mathrm{K}^{-4}$

- Rydberg constant $R_{\infty} \approx 10973731.568508(65) \mathrm{m}^{-1}$

\subsection{Our Model of the Universe}

In 1929, Hubble showed that the universe is expanding [7]. In 1931, Lemaitre was the first to advance the idea that the universe began with a "primeval-atom" [8] which was later ironically nicknamed by Hoyle "Big Bang" in a BBC broadcast in 1949 [9]. According to our model, the material universe is embedded in a luminous universe, both being spherical and expanding with a speed proportional to their radius. The equations of relativity show that any mass must move at a speed lower than $\mathcal{c}$, otherwise it would have infinite energy [10]. Therefore, the expansion of the material universe is slower than its luminous counterpart which is in expansion at the speed of light (which is $c$ for now). With this model, we will calculate some parameters.

With special relativity, Einstein showed that a gravitational field generated by a mass $m$ slows down light [11]. Erroneous by a factor of 2 compared to what happens in reality, his equation, which was coming from special relativity, is subsequently corrected by Schwarzschild using general relativity [12].

We want to show that the speed of light in vacuum may vary in a gravitational field. In their Appendix A, Equation (A.1), Binney and Merrifield cited an Equation (from Weinberg's 1972 book "Gravitation and Cosmology") which gives the proper time $\tau$ for a photon (the same time then if we were traveling on a photon) [13]. We consider this equation as being an excellent approximation (in a context of a weak gravitational field) deduced from general relativity.

$$
\tau^{2}=\left(c^{2}+2 \Phi\right) \mathrm{d} t^{2}-\left(1-2 \Phi / c^{2}\right)\left(\mathrm{d} x^{2}+\mathrm{d} y^{2}+\mathrm{d} z^{2}\right) \quad \text { where } \Phi=\frac{-G m}{r} \leq 0
$$

The $\Phi$ value represents the Newtonian gravitational potential. In a weak gravitational field condition, $|\Phi| \ll c^{2}$. The gravitational potential $\Phi$ is measured at a distance $r$ from the center of mass $m$. Let $\mathrm{d} x, \mathrm{~d} y, \mathrm{~d} z$ be the change in 
the spatial coordinates of a photon in the element of time $\mathrm{d} t$. For an observer at rest, $\tau=0$. Let us consider an arbitrary point $A$ in space which has the spatial coordinate $\left(x_{1}, y_{1}, Z_{1}\right)$. Let us also consider that the center of mass for the mass $m$ is the origin $\mathrm{O}$. We are interested in the gravitational field effect on the refractive index of the vacuum between the point $\mathrm{A}$ and $\mathrm{O}$. We want to calculate the speed of light $v_{L}(r)$ in vacuum for a distance $r=\overline{\mathbf{A O}}$ from the center of mass $\mathrm{O}$. We always have the possibility to make translations and rotations such a way our coordinate system is centered on the point-mass $m$ and that the $x$ axis coincides on the $\overline{\mathbf{A O}}$ line segment. At that time, we can consider that there is no more interaction in other axis. So, $\mathrm{d} y=0$ and $\mathrm{d} z=0$. We then simplify Equation (1) to get Equation (2).

$$
\begin{gathered}
\left(c^{2}+2 \Phi\right) \mathrm{d} t^{2}-\left(1-2 \Phi / c^{2}\right) \mathrm{d} x^{2}=0 \\
v_{L}(r)=\frac{\mathrm{d} x}{\mathrm{~d} t}=\frac{c}{n_{0}} \text { where } n_{0}=\sqrt{\frac{\left(1-2 \Phi / c^{2}\right)}{\left(1+2 \Phi / c^{2}\right)}} \approx 1-2 \Phi / c^{2}
\end{gathered}
$$

For a distance $r$ from the center of mass $m$, the speed of light $c$ is reduced by the refractive index $n_{0}$ caused by a gravitational field of potential $\Phi$, which gives a modified speed of light $v_{L}(r)$. When the gravitational potential $|\Phi| \ll c$ in Equation (3), we can use an approximation for the refractive index $n_{0}$. However, we will not use this approximation in our further calculations.

Locally, in space and time, the speed of light seems constant and equal to $c$. If the universe is expanding and its density is decreasing, the refractive index would also be decreasing, which would imperceptibly accelerate light.

However, the value of $c$ is already the result of another speed limit that we call $k$ (which we do not yet know the value) affected by a local refractive index $n$. Let us use Equation (3) to build another equation with the same form. We are currently at an arbitrary distance $r_{u}$ from the center of mass of the universe. Knowing that the apparent mass of the universe is $m_{u}$, the speed $c$ is the result of Equation (4).

$$
c=\frac{k}{n} \text { where } n=\sqrt{\frac{1-2 \Theta / k^{2}}{1+2 \Theta / k^{2}}} \text { and } \Theta=\frac{-G m_{u}}{r_{u}} \leq 0
$$

The radius of the space [8] [14] (or the radius of the universe [14]), as described by Lemaitre, corresponds to what we prefer to call the apparent radius of curvature of the luminous universe $R_{u}$. We call its value "apparent" because the equation giving $R_{u}$ assumes a speed of light that is constant over time and equal to $c$, for a time equal to the apparent age of the universe $T_{u}=1 / H_{0}$. However, in our model, the speed of light is not constant over time. Its value is indeed $c$ in the present moment. However, assuming that the universe is expanding according to Equation (4), its value was necessarily lower than this speed in the past. If we go back far enough in time (when the apparent radius of curvature of the universe was smaller), we even find a moment where the speed of light was zero.

According to Hubble, the constant $H_{0}$ represents the speed of movement of 
galaxies (in $\mathrm{km} \cdot \mathrm{s}^{-1} \cdot \mathrm{MParsec}$ ) [7]. In our opinion, it is equivalent to locally measuring the derivative of the velocity of matter $V_{m}$ with respect to distance $r$. According to the equations of relativity, to avoid needing infinite energy, matter is obliged to travel slower than light since the speed of light is a speed limit. Matter moves locally at a rate $\beta$ times slower than the speed of light $c$ by moving away radially from the center of mass of the universe. Locally, at our location in the universe, the value of $H_{0}$ is evaluated at a distance $r=r_{u}$ (which represents a fraction $\beta$ of the apparent radius of curvature of the luminous universe $R_{u}$ ).

$$
H_{0}=\left.\frac{\mathrm{d} v_{m}}{\mathrm{~d} r}\right|_{r=r_{u}}=\frac{\beta c}{r_{u}}=\frac{\beta c}{\beta R_{u}} \Rightarrow R_{u}=\frac{c}{H_{0}}
$$

We are at a distance $r_{u}$ from the center of mass of the universe.

$$
r_{u}=\beta R_{u}=\frac{\beta c}{H_{0}}
$$

Let us find the apparent mass of the universe $m_{u}$. Let us also associate a mass $m_{p h}$ with a photon. If we place this photon at the periphery of the luminous universe, at a distance $R_{u}$ from the center of mass of the universe, it will have an $E_{g}$ gravitational energy.

$$
E_{g}=\frac{G m_{u} m_{p h}}{R_{u}}
$$

According to the special relativity, the mass energy associated with this photon is $E_{m}$.

$$
E_{m}=m_{p h} c^{2}
$$

By equating Equation (7) and Equation (8), replacing $R_{u}$ with Equation (5), and isolating the apparent mass of the universe $m_{u}$, we obtain the same equation as that of Carvalho [15].

$$
m_{u}=\frac{c^{2}}{G \cdot R_{u}}=\frac{c^{3}}{G \cdot H_{0}}
$$

For a distance $r_{u}$, our local universe parcel travels at speed $V_{m}$.

$$
v_{m}=\frac{\beta k}{n} \text { where } n=\sqrt{\frac{1-2 \Theta / k^{2}}{1+2 \Theta / k^{2}}} \text { and } \Theta=\frac{-G m_{u}}{r_{u}}
$$

The measurement of $H_{0}$ is made by observing the global displacement of galaxies at our location $r_{u}$. Each galaxy has its own movement. Due to the expansion of the universe, the galaxies are moving away from each other. The value of the Hubble constant $H_{0}$ represents the derivative of the speed of the material universe $V_{m}$ with respect to the element of distance $d r$, evaluated at a distance $r=$ $r_{u}$ from the center of mass of the universe.

$$
H_{0}=\left.\frac{\mathrm{d} v_{m}}{\mathrm{~d} r}\right|_{r=r_{u}}=\frac{k \beta y}{r_{u}}\left(\frac{1}{(1+y) \sqrt{1-y^{2}}}\right) \text { where } y=\frac{2 G m_{u}}{k^{2} r_{u}}
$$

Solving Equations (4) to (6) and Equation (11) leads to Equations (12) to (16). 


$$
\begin{gathered}
k=c \sqrt{2+\sqrt{5}} \approx 6.17 \times 10^{8} \mathrm{~m} / \mathrm{s} \\
\beta=3-\sqrt{5} \approx 0.764 \\
R_{u} \approx 1.28 \times 10^{26} \mathrm{~m} \\
r_{u} \approx 9.80 \times 10^{25} \mathrm{~m} \\
m_{u} \approx 1.73 \times 10^{53} \mathrm{~kg}
\end{gathered}
$$

The $k$ constant represents the asymptotic value for the speed of light in vacuum when the apparent radius of curvature of the universe tends towards infinity. The $\beta$ constant is geometric and can be defined as the ratio between the speed of expansion of the material universe and the speed of expansion of the luminous universe (which is the speed of light). It can also represent the ratio of the apparent radius of curvature of the material universe $r_{u}$ (evaluated at our location in the universe with respect to the center of mass of the universe) and the apparent radius $R_{u}$ of curvature of the light universe. The value of $m_{u}$ represents the apparent mass of the universe.

We draw the reader's attention to the fact that $\beta$ constant is unique to our cosmological model, but it is essential for making multiple connections between physics' constants. It allows making several links between the infinitely large and the infinitely small in Dirac's large numbers hypothesis.

\subsection{Dirac's Large Number Hypothesis}

In this section, we show many equations. In order to condense the information, we ask the reader to refer to the section at the beginning of this article which defines the different parameters of the universe.

By calculating ratios of quantities having the same units, Dirac found that these appeared to result from a few large numbers. Without using all of Dirac's examples, here are some ratios that give the same unitless large number [2].

$$
\frac{R_{u}}{L_{p}}=\frac{m_{u}}{m_{p}}=\frac{m_{p}}{m_{p h}}=\frac{1}{t_{p} H_{0}} \approx 7.94 \times 10^{60}
$$

With similar findings, Dirac figured this was no coincidence. We will see that without $\beta$ in Equation (13), it is impossible to make certain ratios equal to other large numbers.

Dirac could see that these large numbers were separated into a few distinct orders of magnitude without being able to calculate them precisely. All ratios that we found may, by adding certain factors, come from a single number $N$. The $N$ value represents the maximum number of photons having the lowest energy (of $2 \pi R_{u}$ wavelength) that may exist in the universe. Here, we assume that we convert the entire mass $m_{u}$ of the universe in this specific wavelength of photon type. To obtain $N$, let us calculate the mass $m_{p h}$ associated with the $2 \pi R_{u}$ wavelength photon by making the corpuscular and wave energy of a photon equal in Equation (18). 


$$
\begin{gathered}
m_{p h} c^{2}=\frac{h c}{2 \pi R_{u}} \Rightarrow m_{p h}=\frac{h}{2 \pi R_{u} c} \approx 2.74 \times 10^{-69} \mathrm{~kg} \\
N=m_{u} / m_{p h} \approx 6.3 \times 10^{121}
\end{gathered}
$$

The other "large" numbers are of type $N$ exponent a fractional number, such as $N^{1 / 2}, N^{1 / 3}, N^{1 / 4}, \ldots, N^{1 / 57}$ or $N^{2 / 3}, N^{\beta / 4}$, etc. It is possible to find more than a hundred equations giving $N$ itself. Here are a few examples (Equations (20) to (27)) which will be calculated from, among others, postulates \#1 and \#2 cited further. In the following equations, $T_{p}$ represents the Planck temperature which is about $T_{p} \approx 1.42 \times 10^{32} \mathrm{~K}$. This is the highest temperature that can be reached in the universe when we condensate the apparent mass of the universe $m_{u}$ in a point-like sphere of radius equal to Planck length $L_{p}$. We also think this was the initial temperature of the universe at the Big Bang. The value of $q_{p}$ is the Planck charge which is about $q_{p} \approx 1.88 \times 10^{-18} \mathrm{C}$.

$$
\begin{gathered}
N^{1 / 2}=\frac{m_{p}}{m_{p h}}=\frac{R_{u}}{L_{p}}=\frac{1}{t_{p} H_{0}}=\frac{2 \pi T_{p} k_{b}}{h H_{0}}=\frac{-1}{q_{e}} \sqrt{\frac{4 \pi m_{u} R_{u} \alpha}{\mu_{0}}} \approx 7.94 \times 10^{60} \\
N^{1 / 3}=\frac{m_{u} r_{e} \alpha}{m_{e} R_{u} \beta}=\frac{m_{e} \sqrt{\beta}}{m_{p h} \alpha}=\frac{R_{u} \sqrt{\beta}}{r_{e}}=\frac{q_{e}^{2} \alpha}{4 \pi \varepsilon_{0} G \beta m_{e}^{2}} \approx 3.98 \times 10^{40} \\
N^{2 / 3}=\frac{m_{u} \alpha}{m_{e} \beta^{1 / 2}}=\frac{R_{u}^{2} \beta}{r_{e}^{2}}=\frac{m_{p}^{4} \alpha^{4}}{m_{e}^{4} \beta^{2}}=\frac{m_{e}^{2} \beta}{m_{p h}^{2} \alpha^{2}} \approx 1.58 \times 10^{81} \\
N^{1 / 4}=\frac{T_{p}}{T}\left(\frac{15 \beta^{4} \alpha^{2}}{\pi^{3}}\right)^{1 / 4}=\frac{k_{b} T}{m_{p h} c^{2}}\left(\frac{\pi^{3}}{15 \beta^{4} \alpha^{2}}\right)^{1 / 4} \approx 2.82 \times 10^{30} \\
N^{1 / 6}=\frac{r_{e}}{L_{p} \sqrt{\beta}}=\frac{m_{p} \alpha}{m_{e} \sqrt{\beta}}=\frac{\alpha^{3}}{4 \pi R_{\infty} L_{p} \sqrt{\beta}}=\frac{2 \pi r_{e} k_{b} T_{p}}{h c \sqrt{\beta}} \approx 1.99 \times 10^{20} \\
N^{1 / 16}=\left(\frac{4 \pi R_{\infty} c \sqrt{\beta}}{H_{0}}\right)^{57 / 256}=\left(\frac{T_{p} \beta}{T}\right)^{1 / 4}\left(\frac{15 \alpha^{2}}{\pi^{3}}\right)^{1 / 16} \approx 4.10 \times 10^{7} \\
N^{1 / 57}=\frac{q_{p}^{2}}{q_{e}^{2}}=\left(\frac{m_{p}^{2}}{m_{e}^{2} \beta}\right)^{1 / 21}=\left(\frac{q_{e}^{2}}{4 \pi \beta \varepsilon_{0} G m_{e}^{2}}\right)^{1 / 20}=\frac{1}{\alpha} \approx 137 \\
N^{1 / 19}=\left(\frac{m_{e}^{2} \beta}{m_{p h}^{2}}\right)^{1 / 12}=16 \pi^{2} \beta L_{p} R_{u} R_{\infty}^{2} \sqrt{\alpha} \approx 2.57 \times 10^{6} \\
R_{\infty} r_{e}
\end{gathered}
$$

Equation (27) which implies the fine-structure constant $\alpha$ will be used in Equation (48). For rational exponents of small values such as $1 / 57$, we consider the term "large number" is no longer appropriate. Note that our constant $\beta$ is required in several equations.

\subsection{Variations of Physics "Constants" over Time}

According to our model, light accelerates over time. In order for the principle of conservation of energy to be maintained, an electromagnetic wavelength equal to $2 \pi R_{u}$ must increase its wavelength over time to allow light to accelerate during 
the same period. As the universe is expanding, the mass associated with the photon decreases over time.

The universe expands and its angular velocity of rotation decreases like a skater who extends his arms. The radius $R_{u}$ is limited by its tangential rotation speed which is that of light. As the universe is expanding, the refractive index of the vacuum decreases and allows a slow acceleration of light over time.

Photons may have different wavelengths. Therefore, they may have different energy and different associated masses. Let's suppose that we convert all the mass $m_{u}$ of the universe into a huge number $N$ of photons, all having the lowest energy possible. Due to the Plank Equation (see Equation (50) further in this article) used to convert wavelength to energy, these photons are at their lowest energy possible when they will all have the longest wavelength (when $\lambda=2 \pi R_{u}$, which corresponds to the circumference of the universe). It is impossible to have a dimension bigger than the circumference of the universe, thus confirming these photons cannot have any lower energy. Of course, the apparent radius $R_{u}$ of the luminous universe is always increasing over time. Therefore, the mass $m_{p h}$ associated to the energy of one of these photons is decreasing over time since the circumference of the universe will increase in the same time frame. However, in percentage, it will be the same for the mass $m_{u}$ of the universe.

The maximum number $N$ of lowest energy photons is forced to be constant over time since if the mass $m_{p h}$ associated with the lowest energy photons as the denominator of Equation (19) decreases over time, it will be the same, in percentage, for $m_{u}$ which includes the mass of these photons as the numerator. If $N$ is constant, all other large numbers are also constant. In these unitless numbers, the variations in the numerators are compensated by the same percentage changes in the denominators. All "constants" with units though, must vary over time.

Attention, in metrology, it is very useful to consider constant the speed of light. This makes it possible to "freeze" several parameters of our universe and to determine them more precisely. We have every right to do this and to refer all units of measurement to the speed of light, which varies very little in the course of human life. By doing this, we force the fine-structure constant to look like varying over time (even if it is not the case).

If we are trying to make comparisons, it's like having the right to say that a rocket takes off from the ground or to say that the earth is moving under the rocket. We may feel that we have a choice. However, in fact, only one option represents the reality, otherwise, we face the "twin paradox" that was presented to Einstein by Paul Langevin at the Bologna Congress in 1911 (at that time, it was not yet clearly a paradox). Because of the massive mass of the earth and the principle of conservation of the momentum, we are obliged to say that the physical phenomena will be explained only if we consider that the rocket takes off from the ground (and not the opposite). It's the same with the speed of light. To explain physics phenomena, we are forced to admit that the speed of light increases over time. However, for physicists in metrology, it would be a disaster to 
admit such an affirmation since nothing would be really constant anymore, apart from unitless ratios, the fine-structure constant, and the geometrical constants.

By imposing the constancy of $c$ in metrology, certain phenomena become difficult to explain. The expansion of the universe observed by Hubble is incompatible with the "constancy" of $c$ imposed in metrology. If light really accelerates over time and if we continue to say that the speed of the light is constant, that is to say, that the units of distance measure enlarge and that the objects shrink over time. Such considerations may lead some to conclude, erroneously, to the existence of a "Big Crunch". If it is really constant, it is the expansion of the universe that is no longer understandable. Schwarzschild's explanation, with the change in the index of refraction of the vacuum in presence of a mass, would no longer be transposable to the universe as we have done. It would then be necessary to question all calculations made on black holes and gravitational lenses. We cannot believe such an avenue.

Our choice is to see the speed of light accelerating slowly over time. Doing so, all "constants" that have units of measurement vary. Only the unitless constants, such as the fine-structure constant, the unitless ratios and the geometric constants are really constant.

Results will be diametrically opposed if it is taken for granted that the speed of light is constant for metrological purposes or that it changes over time. Both points of view are valid and have their advantages. In this article, it will be considered that the speed of light changes over time.

Einstein's general relativity explains the laws of gravitation by space-time deformations caused by the masses involved [16]. It is however possible to explain the phenomenon of gravitation by a pressure differential caused by a shield effect between different masses located in the vacuum of the universe which is filled with corpuscles transmitting their momentum as described by Fatio [17] and Le Sage [18]. Besides, the Jérôme brothers seem to have succeeded in unifying this concept with Einstein's general relativity [19] (unpublished document). In this scenario, masses would not be attracted, but they would rather be pushed towards one another by invisible corpuscles. According to Sidharth, these corpuscles consist of harmonic oscillators (photons) of different wavelengths [20]. The repeated impacts of photons traveling in all directions in the vacuum of the universe would create a "radiation pressure" (expression used by Mansuripur [21]) or a "thermodynamic radiation pressure" (to refer to the analogous "thermodynamic pressure" term used by Horowitz for gases [22]). Just like in gases where molecules are agitated, the word "thermodynamics" is used to refer to the random stirring of photons. Sidharth considers that the spectrum of these photons' wavelength varies between the Planck length $L_{p}$ and the circumference of the universe $\left(2 \pi R_{u}\right)$. To be more precise, we rather think that these wavelengths vary between the circumference of Planck particle $\left(2 \pi L_{p}\right)$ and the apparent circumference the luminous universe $\left(2 \pi R_{u}\right)$. 
The equations of Einstein's general relativity [16] and Newton's universal gravitation [23] assume that the universal gravitational constant $G$ is constant through universe and through time. But, considering the gravitational force as the result of a radiation pressure, it is possible to conceive that $G$ is constant only for a small parcel of the universe. It's like the water pressure around a fish in the bottom of a lake. The pressure is almost uniform around the fish, but it differs from the surface of the lake. The universe can never end in a "Big Crunch" since the external radiation pressure of it is zero. This pressure deficit generates an irremediable expansion of the universe.

\subsection{First Calculation of a More Precise Hubble Constant}

According to different sources, $H_{0}$ is between $67.8(9) \mathrm{km} \cdot \mathrm{s}^{-1} \cdot \mathrm{MParsec}^{-1}$ [24] and $77.6_{-4.3}^{+4.8} \mathrm{~km} \cdot \mathrm{s}^{-1} \cdot \mathrm{MParsec}^{-1}$ [25]. Uncertainties from different measurement results do not always overlap. For a better precision in calculations that we will do further, we must find a method which will assure us a minimum of unequivocal precision.

As the $\mathrm{CMB}$ average temperature $T$ of the universe can be precisely measured, an exploitable link may be made between this parameter and $H_{0}$.

According to Alpher, the universe has all properties of a black body [26]. The universe absorbs perfectly all electromagnetic energy, whatever wavelengths it receives. This absorption is converted into thermal agitation which causes the emission of a thermal radiation whose emission spectrum on the surface of the sphere of the luminous universe depends only on its average temperature. The Stefan-Boltzmann law allows us to determine the flux density $M^{\circ}\left(\right.$ in $\mathrm{W} \cdot \mathrm{m}^{-2}$ ) as a function of the surface temperature $T$ (in K).

$$
M^{\circ}(T)=\sigma T^{4}
$$

The Stefan-Boltzmann's constant is defined by $\sigma$ in the following equation.

$$
\sigma=\frac{2 \pi^{5} k_{b}^{4}}{15 h^{3} c^{2}} \approx 5.67 \times 10^{-8} \mathrm{~W} \cdot \mathrm{m}^{-2} \cdot \mathrm{K}^{-4}
$$

The flux density on the surface of the sphere representing the luminous universe may be defined as the total power $P_{u}$ dissipated in the universe over the total area $A_{u}$ of the sphere. The dissipated power $P_{u}$ corresponds to taking the total energy $E_{u}$ and dividing it by the apparent age of the universe $T_{u}=1 / H_{0}$.

$$
M^{\circ}=\frac{P_{u}}{A_{u}}=\frac{E_{u}}{A_{u} T_{u}}=\frac{E_{u} H_{0}}{A_{u}}
$$

The total energy of a mass $m$ in movement is given by $E_{t}$.

$$
E_{t}=m c^{2}
$$

The apparent mass $m_{u}$ of the universe described in Equation (9) already represents that of the expanding universe. So, in Equation (31), we replace $m$ by the apparent mass of the universe $m_{u}$ and the energy $E_{t}$ by the total amount of energy $E_{u}$ contained in the universe. 


$$
E_{u}=m_{u} c^{2}=\frac{c^{5}}{G H_{0}}
$$

Using Equations (28) to (30), and Equation (32), we get the value of $T$ which should be the average temperature of the Cosmic Microwave Background (CMB).

$$
T=\frac{1}{k_{b}}\left(\frac{15 h^{3} c^{7}}{2 \pi^{5} A_{u} G}\right)^{1 / 4}
$$

Ludwig Boltzmann expressed the statistical entropy $S$ [27] as a function of the number $\Omega$ of microstates defining the equilibrium of a given macroscopic system.

$$
S=k_{b} \ln (\Omega)
$$

The expansion of the material universe is $\beta$ times slower than that of the luminous universe. Entropy is a measure of disorder in the universe and increases with the expansion rhythm. Its measure at $R_{u}$ would have the value $S^{\prime}$.

$$
S^{\prime}=\frac{S}{\beta}=\frac{k_{b} \ln (\Omega)}{\beta}=k_{b}^{\prime} \ln (\Omega) \text { where } k_{b}^{\prime}=\frac{k_{b}}{\beta}
$$

The "Boltzmann constant" $k_{b}$ is true locally in our universe, at our position $r_{u}$ with respect to the center of mass of the universe. At the periphery of the luminous universe, at $R_{u}=r_{u} / \beta$, the "Boltzmann constant" becomes $k_{b}^{\prime}$ as given in Equation (35). At the periphery of the luminous universe, Equation (33) becomes Equation (36).

$$
T=\frac{1}{k_{b}^{\prime}}\left(\frac{15 h^{3} c^{7}}{2 \pi^{5} A_{u} G}\right)^{1 / 4}=\frac{\beta}{k_{b}}\left(\frac{15 h^{3} c^{7}}{2 \pi^{5} A_{u} G}\right)^{1 / 4}
$$

Let's find the $A_{u}$ area of the sphere of the luminous universe when the universe is static.

$$
A_{u}=4 \pi R_{u}^{2} \text { (For static universe) }
$$

Applying this assumption to Equation $(36), T \approx 31.9 \mathrm{~K}$. This is false because with the Cobra probe $T \approx 2.736(17) \mathrm{K}$ [5]. It is like if the volume of the static universe were not big enough to dissipate the energy down to the desired value. However, according to Hawking [28] and Fennelly [29], the universe is rotating. Einstein showed that a rotating disk has a larger circumference than a static disk [30]. The circumference becomes "Cir.".

$$
\text { Cir. }=2 \pi R_{u}^{\prime}=\frac{2 \pi R_{u}}{\sqrt{1-v^{2} / c^{2}}} \Rightarrow R_{u}^{\prime}=\frac{R_{u}}{\sqrt{1-v^{2} / c^{2}}} \text { (Universe in rotation) }
$$

We think that the tangential speed of rotation of the universe is the same as the tangential speed of rotation of the electron on itself. As Llewellyn [31], let us suppose that the spin of the electron is caused by a rotation of the electron on itself.

As it will be shown in Equation (53) and Equation (56), the mass $m_{e}$ and the charge $q_{e}$ of the electron are contained within the classical radius $r_{e}$ of the elec- 
tron. The wave energy associated with the electron is, for its part, contained in the Compton radius $r_{c}$ which is $1 / \alpha \approx 137$ times larger than $r_{e}$. A wave can be seen in two different ways. From the side, it will look like a sinusoid. But seen from the front, it looks like a circle. As for an electromagnetic wave, the frequency of the wave associated with the electron is calculated by considering that the circumference of the wave circle is traveling at the speed of light.

There seems to be only one way to explain the fact that the mass of the electron is contained in the radius $r_{e}$ and that its wave energy is contained in the Compton radius $r_{c}$. This is because the mass $m_{e}$ of the electron turns at a relativistic speed such that its Lorentz factor is equal to the fine-structure constant $\alpha$, which would explain why $r_{c}=r_{e} / \alpha$.

$$
\alpha=r_{e} / r_{c}=\sqrt{1-v^{2} / c^{2}}
$$

We make the hypothesis that the tangential velocity $v$ of the luminous universe periphery is the same as that of the electron.

$$
v=c \sqrt{(1-\alpha)(1+\alpha)} \approx 0.999973 c
$$

This speed is close to that of light. For an observer located at the center of rotation, the time that elapses in the periphery of the luminous universe is dilated and the distances are compressed by the Lorentz factor. Equation (38) becomes Equation (41).

$$
R_{u}^{\prime}=R_{u} / \alpha \quad \text { (Universe in rotation) }
$$

The area of the outer surface of the sphere of our universe from Equation (37) becomes equal to Equation (42).

$$
A_{u}=4 \pi R_{u}^{2} / \alpha^{2} \quad \text { (Universe in rotation) }
$$

with Equation (5) and Equation (42), we modify Equation (36) to obtain Equation (43) which should give the average cosmological microwave background (CMB) temperature $T$.

$$
T=\frac{\beta}{k_{b}}\left(\frac{15 \alpha^{2} h^{3} c^{5} H_{0}^{2}}{8 \pi^{6} G}\right)^{1 / 4}
$$

By varying $H_{0}$ between 67.8 and $77.6 \mathrm{~km} \cdot \mathrm{s}^{-1} \cdot \mathrm{MParsec}^{-1}$ of the Planck [24] and Chandra [25] probes respectively, we obtain a value of $T$ between $2.65 \mathrm{~K}$ and 2.84 K. Data from Cobra (2.736(17) K [5]) and WMAP (2.72548(57) K [6]) probes confirm these results with a lower uncertainty than that currently weighing on $H_{0}$.

Let Equation (43) being equal to Fixsen's $T$ and isolating $H_{0}$ to calculate its value.

$$
H_{0}(T)=\frac{\pi^{3} T^{2} k_{b}^{2}}{\beta^{2} \alpha} \sqrt{\frac{8 G}{15 c^{5} h^{3}}} \approx 71.505(30) \mathrm{km} \cdot \mathrm{s}^{-1} \cdot \mathrm{MParsec}^{-1}
$$

Equation (44) agrees with Salvatelli who gets $H_{0} \approx 72.1_{-2.3}^{+3.2} \mathrm{~km} \cdot \mathrm{s}^{-1} \cdot \mathrm{MParsec}^{-1}$ [4]. It is almost the average result between that of the Planck and Chandra probes. 


\subsection{Hypothesis Leading to an Exact Calculation of the Universal Gravitational and Hubble Constants}

We assume that the $\alpha$ constant can be used as a scale factor. Applied a certain number of times, this factor can be related to some unitless ratios involving physics constants.

$$
m_{u}=m_{p h} / \alpha^{n}
$$

Let us start by incorporating Equation (5), Equation (18), Equation (44) and Equation (45) into Equation (19) in order to find $N$ and then $n$. The accuracy of $N(T, G)$ will especially depend on the temperature $T$ that will be used in Equation (46) and on the universal gravitational constant $G$. Any little change in other parameters (within the tolerances mentioned in the CODATA) of Equation (46) will not have any impact on the result value since $T$ and $G$ are much less accurate than the others that are used in this equation. Let's take $T \approx 2.72548$ (57) $\mathrm{K}$ from Fixsen coming from the Wilkinson Microwave Anisotropic Probe (WMAP) [6]) and $G \approx 6.67408(31) \times 10^{-11} \mathrm{~m}^{3} \cdot \mathrm{kg}^{-1} \cdot \mathrm{s}^{-2}$ from the CODATA 2014 [3].

$$
N(T, G)=\frac{1}{\alpha^{n}}=\frac{m_{u}}{m_{p h}}=\frac{15 \alpha^{2} \beta^{2} h^{2} c^{10}}{4 \pi^{5} T^{4} k_{b}^{4} G^{2}} \approx 6.4071(54) \times 10^{121}
$$

Let us define $[x]$ as being the rounded integer value of $x$.

$$
n=[-\log (N) / \log (\alpha)]=[57.00332(17)]=57
$$

For now, this result cannot be demonstrated, we must create the postulate \#1.

$$
\text { POSTULATE \#1: } \quad N=1 / \alpha^{57} \approx 6.303419702(84) \times 10^{121}
$$

\subsection{Calculation of the Universal Gravitational Constant}

To calculate $G$, we need an equation giving exactly the value $N$ obtained by Equation (48) as a function of $G$, but independent of $H_{0}$ and $T$ that we do not know with sufficient precision (compared to other physics constants from CODATA).

The most frequent opportunities to see the constant $G$ intervene in physics equations are found in the calculations of gravitational energy and gravitational force. Let us start by evaluating, on Earth, the gravitational energy $E_{g}$ that there is between two electrons separated by a distance equal to the classical radius $r_{e}$ of the electron.

$$
E_{g}=\frac{G m_{e}^{2}}{r_{e}}
$$

The energy of an electromagnetic wave of wavelength $\lambda$ is given by Equation (50).

$$
E=\frac{h c}{\lambda}
$$

Even in a context where the speed of light increases over time, the principle of 
conservation of energy must be respected. For this reason, the photons that are on the periphery of the luminous universe continue to see their wavelength increase over time to gradually adapt to the new diameter of the universe. The same goes for all other lengths in the universe. We do not realize this because all the other physics constants change at the same time (except unitless ratios, the fine-structure constant and the geometric constants).

Let us bring the experiment made with the two electrons to the periphery of the luminous universe. By doing this, the classical radius of the electron will increase by a factor $\beta$ and become $r_{e}^{\prime}$ (just as for the apparent radius of curvature of the universe).

$$
r_{e}^{\prime}=\frac{r_{e}}{\beta}
$$

The gravitational energy of Equation (49) is now given by Equation (52).

$$
E_{g}^{\prime}=\frac{G m_{e}^{2}}{r_{e}^{\prime}}=\frac{G m_{e}^{2} \beta}{r_{e}}
$$

On the other hand, the electrical energy $E_{e}$ remains the same whether measured here on Earth or at the outskirts of the universe. This equation is independent of the radius (although it seems to depend on it at first). Let us analyze the equation of $E_{e}$ on Earth.

$$
E_{e}=\frac{q_{e}^{2}}{4 \pi \varepsilon_{0} r_{e}}
$$

The charge $q_{e}$ of an electron is obtained by Equation (54).

$$
q_{e}=-\sqrt{\frac{4 \pi m_{e} r_{e}}{\mu_{0}}}
$$

The speed of light $c$ is related to $\varepsilon_{0}$ and $\mu_{0}$ by Equation (55).

$$
c=1 / \sqrt{\varepsilon_{0} \mu_{0}}
$$

If we use Equation (54) and Equation (55) in Equation (53), we obtain Equation (56).

$$
E_{e}=m_{e} c^{2}
$$

Equation (53) and Equation (56) show that the energy contained in the mass of an electron is equivalent to the electrical energy contained between two electrons spaced with a distance equivalent to the classical radius of the electron $r_{e}$. In Equation (56), it can be seen that the electric energy $E_{e}$ is independent of the classical radius of an electron. Therefore, if we reproduce this experience on the periphery of the luminous universe, the electric energy $E_{e}^{\prime}$ that we will have will be equal to the energy $E_{e}$ that we had on Earth. Let us then make the connection between the electric energy $E_{e}^{\prime}$ and the gravitational energy $E_{g}^{\prime}$ at the periphery of the luminous universe. Bringing the experiment to the periphery of the luminous universe allows seeing why a $\beta$ factor is introduced into the equation. 


$$
\frac{E_{e}^{\prime}}{E_{g}^{\prime}}=\frac{m_{e} c^{2} r_{e}}{G m_{e}^{2} \beta}=\frac{c^{2} r_{e}}{G m_{e} \beta} \approx 5.45 \times 10^{42}
$$

As in Equation (48), we found that the fine-structure constant $\alpha$ plays a role in determining orders of magnitude. By an adjustment of the exponent of the fine-structure constant $\alpha$, we obtain a result identical to that of Equation (57).

$$
\frac{1}{\alpha^{20}} \approx 5.45 \times 10^{42}
$$

We conclude that Equation (57) and Equation (58) are equal. By isolating the universal gravitational constant $G$, we obtain an equation that we elevate to the rank of postulate \#2. It is an equation that cannot be deduced from any other known equation of current physics.

$$
\text { POSTULATE \#2: } \quad G=\frac{c^{2} r_{e} \alpha^{20}}{m_{e} \beta} \approx 6.673229809(86) \times 10^{-11} \mathrm{~m}^{3} \cdot \mathrm{kg}^{-1} \cdot \mathrm{s}^{-2} \text { (59) }
$$

This result is in perfect agreement with the Taylor-Parker-Langenberg value which is $G \approx 6.6732(31) \times 10^{-11} \mathrm{~m}^{3} \cdot \mathrm{kg}^{-1} \cdot \mathrm{s}^{-2}$ [32]. However, based on Equation (59), it can be seen that the tolerance of the measured value of $G$ from CODATA 2014 [3] is underestimated by a factor of about 2.74. Qing [33] has shown that the tolerances of several recent measurements of $G$ do not overlap with each other. Despite the very optimistic accuracy displayed for the various measures, the value of $G$ lies between 6.672 to $6.676 \times 10^{-11} \mathrm{~m}^{3} \cdot \mathrm{kg}^{-1} \cdot \mathrm{s}^{-2}$. The tolerance displayed in Equation (59) was calculated from the tolerances displayed for the other constants of CODATA 2014. When measuring the universal gravitational constant $G$, it is easy to underestimate some sources of errors, which can explain this difference. Equation (59) relies on more precise and reproducible constants.

By using different combinations of equations, shown in this article, we can deduce other equations which also give the universal gravitational constant $G$ precisely. We will enumerate them without making any demonstration.

$$
G=\frac{q_{e}^{2} r_{e} R_{\infty} \alpha^{17}}{\varepsilon_{0} m_{e}^{2} \beta}=\frac{2 \pi c^{3} r_{e}^{2} \alpha^{19}}{h \beta}=\frac{q_{e}^{2} \alpha^{20}}{4 \pi \varepsilon_{0} m_{e}^{2} \beta}=\frac{h c \alpha^{21}}{2 \pi m_{e}^{2} \beta}=\frac{c^{2} \alpha^{23}}{4 \pi R_{\infty} m_{e} \beta}
$$

\subsection{Improvement of the Hubble Constant and of the Average CMB Temperature}

Now that we can precisely calculate $N$ and $G$, we can equate Equation (19) with Equation (48) using Equation (9), Equation (18) and Equation (59).

$$
N=\frac{m_{u}}{m_{p h}}=\frac{2 \pi c^{5}}{h G H_{0}^{2}}=\frac{2 \pi \beta m_{e} c^{3}}{h r_{e} H_{0}^{2} \alpha^{20}}=\frac{1}{\alpha^{57}}
$$

Let's associate the energy of the mass $m_{e}$ of an electron with the wave energy.

$$
m_{e} c^{2}=\frac{h c \alpha}{2 \pi r_{e}}
$$

With Equation (62), we modify Equation (61) to obtain Equation (63). 


$$
H_{0}=\frac{c \alpha^{19} \sqrt{\beta}}{r_{e}} \approx 72.09548580(32) \mathrm{km} \cdot \mathrm{s}^{-1} \cdot \mathrm{MParsec}^{-1}
$$

The result of Equation (63) is similar to Equation (44) with Fixsen and confirms Salvatelli's value of $H_{0} \approx 72.1_{-2.3}^{+3.2} \mathrm{~km} \cdot \mathrm{s}^{-1} \cdot \mathrm{MParsec}^{-1}$. Thanks to various equations in this article, we can deduce new equations which will calculate $H_{0}$ precisely. We will enumerate them without making any demonstration.

$$
H_{0}=4 \pi c R_{\infty} \alpha^{16} \sqrt{\beta}=\frac{4 \pi m_{e} c \alpha^{19} \sqrt{\beta}}{q_{e}^{2} \mu_{0}}=\frac{2 \pi m_{e} c^{2} \alpha^{18} \sqrt{\beta}}{h}
$$

Equating Equation (44) and Equation (63), and using Equation (62), we obtain $T$ which is the average CMB temperature.

$$
T=\frac{m_{e} c^{2}}{k_{b}}\left(\frac{15 \beta^{6} \alpha^{17}}{\pi^{3}}\right)^{1 / 4} \approx 2.7367958(16) \mathrm{K}
$$

\subsection{Weinberg's Formula}

Weinberg found an empirical equation for a typical mass $m$ of a pion-like particle (see page 619 in the Weinberg's 1972 book available in reference [34]). This equation has also been cited by Sidharth in reference [20]. However, the result of this equation does not precisely correspond to any known value. Therefore, we think it is incomplete. It is like if a multiplying factor is missing. In the following equation, $\hbar=h / 2 \pi$.

$$
m \approx\left(\frac{H_{0} \hbar^{2}}{G c}\right)^{1 / 3} \approx 1 \times 10^{-28} \mathrm{~kg}
$$

Let us show that the precise values of $\alpha, \beta, G$ and $H_{0}$ result in the mass $m_{e}$ of an electron from a modified version of Weinberg's Equation (66). Let us multiply Equation (63) by 1 (in parenthesis, let us multiply by $G$ and divide by Equation (59)).

$$
H_{0}=\frac{c \alpha^{19} \sqrt{\beta}}{r_{e}}\left(\frac{G m_{e} \beta}{c^{2} r_{e} \alpha^{20}}\right)=\frac{G m_{e} \beta^{3 / 2}}{c r_{e}^{2} \alpha}
$$

Isolating $m_{e}$, we obtain Equation (68).

$$
m_{e}=\frac{c H_{0} r_{e}^{2} \alpha}{\beta^{3 / 2} G}
$$

The energy contained in the mass $m_{e}$ of an electron at rest is equal to the wave energy for the Compton wavelength $\lambda_{c}$ of the electron.

$$
\begin{gathered}
m_{e} c^{2}=\frac{h c}{\lambda_{c}} \text { where } \lambda_{c}=\frac{2 \pi r_{e}}{\alpha} \Rightarrow m_{e} c^{2}=\frac{h c \alpha}{2 \pi r_{e}} \\
r_{e}^{2}=\frac{\hbar^{2} \alpha^{2}}{m_{e}^{2} c^{2}}
\end{gathered}
$$

Introducing the result of Equation (70) in Equation (68), we obtain the electron mass $m_{e}$ as a function of $G$ and $H_{0}$. Of course, we get the same value than 
the CODATA (except the tolerance), but this equation should not be considered as a way to get the mass of the electron since our values of $G$ and $H_{0}$ come someway from this equation. However, as we will see, Equation (71) may be useful for other purposes.

$$
m_{e}\left(G, H_{0}\right)=\frac{\alpha}{\sqrt{\beta}}\left(\frac{H_{0} \hbar^{2}}{G c}\right)^{1 / 3} \approx 9.109383559(85) \times 10^{-31} \mathrm{~kg}
$$

The apparent age of the universe is $1 / H_{0}$ and Equation (71) is a function of $H_{0}$. As a result, the mass $m_{e}$ of the electron varies with time. Since this is the case for $m_{e}$ it is also the case of other particles and for the apparent mass of the universe $m_{u}$. Without doing it here, it can be shown that this is the case for all other "constants" with units.

Equation (71), which is a modified version of the Weinberg formula, could be used to determine and calculate the Hubble constant $H_{0}$ as a function of the universal gravitational constant $G$ because all other constants of the equation are precisely known.

$$
H_{0}(G)=\frac{G c m_{e}^{3} \beta^{3 / 2}}{\hbar^{2} \alpha^{3}} \approx 72.104(33) \mathrm{km} \cdot \mathrm{s}^{-1} \cdot \text { MParsec }
$$

Using the value of $G \approx 6.67408(31) \times 10^{-11} \mathrm{~m}^{3} \cdot \mathrm{kg}^{-1} \cdot \mathrm{s}^{-2}$ from CODATA 2014 [3] in Equation (72) we obtain a measured value of $H_{0}$ which is similar to the result of Equation (63). This value of $H_{0}$ corresponds to an apparent age of the universe of 13.56(6) billion years. Our $H_{0}$ value from Equation (63) gives also about 13.56 billion years.

\section{Conclusions}

This article seems to be very useful from a metrological point of view since it makes it possible to precisely determine several constants which were, until now, only measured. The equations found make it possible to discriminate the results of several research studies on the Hubble constant, on the universal gravitational constant and on the average CMB temperature.

A new model of the universe that uses the $\beta$ constant has been introduced. This constant represents the ratio between the expansion speed of the material universe and the expansion speed of the luminous universe which is the speed of light. This constant seems to let us believe that the universe is geometric in a certain way. It may be found that $\beta$ constant is essential in the evaluation of several parameters of the universe (see Equation (59), Equation (63), Equation (65), Equation (71), and many others). We have also seen this by using it to modify the Dirac hypothesis on large numbers because it allows making several links that would not be possible without it. This hypothesis allows making an important link with the fine-structure constant $\alpha$. Using this link, we derived equations giving the exact values of $H_{0}, G$ and $T$. Knowing where these parameters come from, we can explain why these parameters of the universe are not constant across space and the time. 
We used a modified version of Weinberg's equation which undoubtedly shows that there is a link between the electron mass, the Hubble constant $H_{0}$ and the universal gravitational constant $G$. This link gives, for the first time, a precise tool to measure the Hubble constant here on Earth, without having to use star observations.

We determined that only unitless parameters are constant. To have a precise metrology system, the International Bureau of Weights and Measures (BIPM) makes the choice to impose the constancy of $c$. This relevant choice makes it possible to increase the precision of the other physics parameters by becoming really constant. Unitless constants, such as $\alpha$, will appear to vary over time and some phenomena will become difficult to explain. To consider or not $c$ as constant seems incompatible without really being so. One must be aware of the choice of hypotheses that are being made in the theoretical analyses of scientific documents. It is important to define the chosen choices from the beginning.

One must be aware of the choice being made in theoretical analyzes. The chosen choices must be defined right at the beginning.

By using the equations of $G$ and $H_{0}$ shown in this article, it will probably be possible to determine more precisely the movement speeds of the different galaxies, their masses and several other parameters of the universe. By using the equation giving $N$ in the modified hypothesis of large numbers of Dirac, it will probably be possible to establish close links between several physics' constants, which will make it possible to determine new equations that were previously unknown to us.

\section{Conflicts of Interest}

The author declares no conflicts of interest regarding the publication of this paper.

\section{References}

[1] Dirac, P.A.M. (1938) Proceedings of the Royal Society of London A: Mathematical, Physical and Engineering Sciences, 165, 199-208. https://doi.org/10.1098/rspa.1938.0053

[2] Dirac, P.A.M. (1974) Proceedings of the Royal Society of London A: Mathematical, Physical and Engineering Sciences, 338, 439-446. https://doi.org/10.1098/rspa.1974.0095

[3] Mohr, P.J., Newell, D.B. and Taylor, B.N. (2016) Journal of Physical and Chemical Reference Data, 45, Article ID: 043102. https://doi.org/10.1063/1.4954402

[4] Salvatelli, V., Andrea, M., Laura, L.-H. and Olga, M. (2013) Physical Review D, 88, Article ID: 023531. https://doi.org/10.1103/PhysRevD.88.023531

[5] Gush, H.P. (1981) Physical Review Letters, 47, 745-748. https://doi.org/10.1103/PhysRevLett.47.745

[6] Fixsen, D.J. (2009) The Astrophysical Journal Supplement Series, 707, 916-920. https://doi.org/10.1088/0004-637X/707/2/916

[7] Hubble, E. (1929) PNAS, 15, 168-173. https://doi.org/10.1073/pnas.15.3.168 
[8] Kragh, H. (2012) Astrophysics and Space Science Library, 395, 23-38. https://doi.org/10.1007/978-3-642-32254-9 3

[9] Kragh, H. (2013) Astronomy \& Geophysics, 54, 28-30. https://doi.org/10.1093/astrogeo/att035

[10] Einstein, A. (1905) Annalen der Physik, 322, 891-921. https://doi.org/10.1002/andp.19053221004

[11] Einstein, A. (1911) Annalen der Physik, 35, 898-908. https://doi.org/10.1002/andp.19113401005

[12] Grøn, Ø. (2016) American Journal of Physics, 84, 537. https://doi.org/10.1119/1.4944031

[13] Binney, J. and Merrifield, M. (1998) Galactic Astronomy. Princeton University Press, Princeton, 816. https://press.princeton.edu/titles/6358.html

[14] Lemaître, G. (2013) General Relativity and Gravitation, 45, 1635-1646. https://doi.org/10.1007/s10714-013-1548-3

[15] Carvalho, J.C. (1995) International Journal of Theoretical Physics, 34, 2507-2509. https://doi.org/10.1007/BF00670782

[16] Einstein, A. (1916) Annalen der Physik, 49, 769-822. https://doi.org/10.4324/9780203198711

[17] Fatio de Duillier, N. (1949) Royal Society of London, 6, 125-160. https://doi.org/10.1098/rsnr.1949.0018

[18] Rolinson, J.S. (2003) Notes and Records of the Royal Society of London, 57, 35-45. https://doi.org/10.1098/rsnr.2003.0195

[19] Jérôme, J. and Jérôme, F. (2012) New Version of General Relativity that Unifies Mass and Gravity in a Common 4D Higgs Compatible Theory. https://www.researchgate.net/publication/279291155 New Version of General Relativ ity that Unifies Mass and Gravity in a Common 4D Higgs Compatible Theory

[20] Sidharth, B.G. (2008) The Thermodynamic Universe. Cornell University, International Institute of Applicable Mathematics \& Information Sciences, World Scientific, Hackensack, 304. https://doi.org/10.1142/6815

[21] Mansuripur, M. and Pin, H. (2017) Thermodynamics of Radiation Pressure and Photon Momentum. Optical Trapping and Optical Micromanipulation, Vol. 14, San Diego, 6-10 August 2017, 20. https://doi.org/10.1117/12.2274589

[22] Horowitz, J. (1950) Journal de Physique et Le Radium, 11, 241. https://doi.org/10.1051/jphysrad:01950001105024100

[23] Newton, I. (1686) Philosophiae Naturalis Principia Mathematica. S. Pepys, Reg. Soc. Praeses, London, 510. https://doi.org/10.5479/sil.52126.39088015628399

[24] Ade, P.A.R., et al. (2016) Astronomy \& Astrophysics, 594, A13. https://doi.org/10.1051/0004-6361/201525830

[25] Bonamente, M., Joy, M.K., La Roque, S.J. and Carlstrom, J.E. (2005) The Astrophysical Journal, 647, 25-54. https://doi.org/10.1086/505291

[26] Alpher, R.A. and Herman, R.C. (1948) Nature, 162, 774-775. https://doi.org/10.1038/162774b0

[27] Sharp, K. and Matschinsky, F. (2015) Translation of Ludwig Boltzmann's Paper “On the Relationship between the Second Fundamental Theorem of the Mechanical Theory of Heat and Probability Calculations Regarding the Conditions for Thermal Equilibrium" Sitzungberichte der Kaiserlichen Akademie der Wissenschaften. Mathematisch-Naturwissen Classe. Abt. II, LXXVI 1877, pp 373-435 (Wien. Ber. 1877, 
76:373-435). Reprinted in Wiss. Abhandlungen, Vol. II, Reprint 42, p. 164-223, Barth, Leipzig, 1909. Entropy, 17, 1971-2009.

https://doi.org/10.1142/9781848162938 0002

[28] Hawking, S. (1969) Monthly Notices of the Royal Astronomical Society, 142, 129-141. https://doi.org/10.1093/mnras/142.2.129

[29] Fennelly, A.J. (1976) The Astrophysical Journal, 207, 693-699. https://doi.org/10.1086/154537

[30] Einstein, A. (1912) Annalen der Physik, 347, 355-369. https://doi.org/10.1002/andp.19123430704

[31] Llewellyn, T. (1926) Nature, 117, 514. https://doi.org/10.1038/117514a0

[32] Taylor, B.N., Parker, W.H. and Langenberg, D.N. (1969) Reviews of Modern Physics, 41, 375-496. https://doi.org/10.1103/RevModPhys.41.375

[33] Qing, L., et al. (2018) Nature, 560, 582-588. https://doi.org/10.1038/s41586-018-0431-5

[34] Weinberg, S. (1972) Gravitation and Cosmology: Principles and Applications of the General Theory of Relativity. John Wiley \& Sons, New York, 685.

https://archive.org/details/WeinbergS.GravitationAndCosmology..PrinciplesAndAp plicationsOfTheGeneralTheoryOf 


\section{Appendix A: Solving of the System of Equations}

In this appendix, we will focus on detailing calculations that made it possible to solve a system of 5 unknowns with 5 equations. We will use Equation (59) to evaluate the universal gravitational constant $G$ and Equation (63) to evaluate the Hubble constant $H_{0}$.

Let us start by rewriting Equation (4) in another form that will be more practical and list the other starting equations.

$$
\begin{gathered}
c=\frac{k}{n} \text { where } n=\sqrt{\frac{1+y}{1-y}} \text { and } y=\frac{2 G m_{u}}{r_{u}} \\
R_{u}=\frac{c}{H_{0}} \\
r_{u}=\frac{\beta c}{H_{0}}=\beta R_{u} \\
m_{u}=\frac{c^{3}}{G H_{0}} \\
H_{0}=\left.\frac{\mathrm{d} v_{m}}{\mathrm{~d} r}\right|_{r=r_{u}}=\frac{\beta k y}{r_{u}}\left(\frac{1}{(1+y) \sqrt{1-y^{2}}}\right) \text { where } y=\frac{2 G m_{u}}{k^{2} r_{u}}
\end{gathered}
$$

In these equations, the unknown values are: $R_{u}, r_{u}, m_{u}, \beta$ and $k$. It is a system of 5 equations with 5 unknowns that can be solved mathematically.

In Equation (73), we isolate the value of $k$ and obtain Equation (78).

$$
k=c \sqrt{\frac{1+y}{1-y}}
$$

In Equation (77), we also isolate the value of $k$ to obtain Equation (79).

$$
k=\frac{H_{0} r_{u}}{y \beta}(1+y) \sqrt{1-y^{2}}
$$

Let us equate Equation (78) and Equation (79) to obtain Equation (80).

$$
c \sqrt{\frac{1+y}{1-y}}=\frac{H_{0} r_{u}}{y \beta}(1+y) \sqrt{1-y^{2}}
$$

Let us use Equation (74) and Equation (75) in Equation (80) and simplify to get Equation (81).

$$
y^{2}+y-1=0 \Rightarrow y=\frac{-1 \pm \sqrt{5}}{2}
$$

All $y$ parameters in Equation (73) are strictly positive. Therefore, $y$ is positive.

$$
y=\frac{\sqrt{5}-1}{2}
$$

Putting the result of (82) in Equation (73), we obtain Equation (83).

$$
k=c \sqrt{2+\sqrt{5}}
$$

In Equation (77), substitute the value of $y$ for the parenthesis by its algebraic 
value.

$$
H_{0}=\frac{2 G m_{u} \beta}{k r_{u}^{2}}\left(\frac{1}{(1+y) \sqrt{1-y^{2}}}\right)
$$

In Equation (84), replace the value of $m_{u}$ with Equation (76).

$$
H_{0}=\frac{2 c^{3} \beta}{k r_{u}^{2} H_{0}}\left(\frac{1}{(1+y) \sqrt{1-y^{2}}}\right)
$$

In Equation (85), we use Equation (75) and Equation (83), then we isolate $\beta$.

$$
\beta=\frac{2}{\sqrt{2+\sqrt{5}}}\left(\frac{1}{(1+y) \sqrt{1-y^{2}}}\right)
$$

In Equation (86), let us replace $y$ by Equation (82) with the $\beta$ value. Then, let us list the other results from our system of equations of and let us evaluate their value.

$$
\begin{gathered}
\beta=3-\sqrt{5} \approx 0.764 \\
k=c \sqrt{2+\sqrt{5}} \approx 2 c \approx 6 \times 10^{8} \mathrm{~m} \cdot \mathrm{s}^{-1} \\
m_{u} \approx 1.8 \times 10^{53} \mathrm{~kg} \\
R_{u} \approx 1.28 \times 10^{26} \mathrm{~m} \\
r_{u} \approx 0.98 \times 10^{26} \mathrm{~m}
\end{gathered}
$$

Let us note that $\beta$ is related to the golden number which is $\varphi \approx 1.618 \ldots$

$$
\beta=4-2 \varphi \text { where } \varphi=\frac{1+\sqrt{5}}{2}
$$

